\title{
Surface models and gradually stripped volume model to explore the foot muscles
}

\author{
Dong Sun Shin ${ }^{1}$, Koojoo Kwon ${ }^{2}$ Byeong-Seok Shin ${ }^{2}$, Hyung Seon Park ${ }^{3}$, Sangho Lee ${ }^{3}$, \\ Seung-Bock Lee ${ }^{3}$, Min Suk Chung ${ }^{4}$ \\ ${ }^{1}$ Department of Orthopaedic Biomaterial Science, Osaka University Graduate School of Medicine, Osaka, Japan \\ ${ }^{2}$ Department of Computer Science and Information Technology, Inha University, Incheon, Republic of Korea \\ ${ }^{3}$ Korea Institute of Science and Technology Information, Daejeon, Republic of Korea \\ ${ }^{4}$ Department of Anatomy, Ajou University School of Medicine, Suwon, Republic of Korea
}

\begin{abstract}
Objectives: Authors intended to confirm whether simultaneous watching of the surface and the volume models contributes to foot anatomy.

Methods: Outlines of the skin and foot muscles were traced in the sectioned images of a cadaver to build surface models of the structures. After the outlines were filled with appointed colors, the sectioned images, including the colors, were stacked to yield the volume model of foot which was stripped from the skin little by little.

Results: The stripped volumes illustrated the depth of the individual foot muscles. In clinics, the stripped volumes of the foot's computed tomographs and magnetic resonance images could be a solution for standardization regardless of the degrees of plantar flexion.

Conclusion: The exploration of volume model, accompanied by equivalent surface models, can potentially be applied to anatomical research in order to discern the morphological properties of a region or an organ.
\end{abstract}

Keywords: computer-assisted image processing; foot; muscles; three-dimensional imaging; Visible Human Projects

Anatomy 2015;9(1):19-25 C2015 Turkish Society of Anatomy and Clinical Anatomy (TSACA)

\section{Introduction}

Unlike computed tomographs (CTs) or magnetic resonance images (MRIs), serially sectioned images of cadavers comprise high resolution and real body color. So the sectioned images enable the segmentation and three-dimensional (3D) reconstruction of detailed structures. ${ }^{[1]}$ The surface models having small file size can be selected to display, can be rotated and modified in real time. ${ }^{[2-5]}$ Comparatively, the volume models with huge voxel information can be arbitrarily cut to show the sections. ${ }^{[6-7]}$ In a recent study with the Visible Korean data, the volume model of ascending colon was stripped at constant thicknesses, creating new features of its wall. ${ }^{[8]}$ The authors chose, as subsequent target, the foot consisting of complicated skeletal muscles.

The aim of this study was to confirm whether various observations of the surface and volume models are benefi- cial to foot anatomy comprehension. To achieve the goal, outlines of the foot muscles were drawn in the sectioned images and stacked to produce surface models. Concurrently, the sectioned images, including the designated colors of outlined structures, were used to yield the volume model of foot, which was stripped from the skin little by little.

\section{Materials and Methods}

In the Visible Korean project, sectioned images (intervals $0.2 \mathrm{~mm}$ ) were acquired from an entire male cadaver (33 years old; statue $1.64 \mathrm{~m}$; weight $55 \mathrm{~kg}$ ) ${ }^{[1]}{ }^{1]}$ We selected 488 images (intervals $0.4 \mathrm{~mm}$ ) from the distal part of tibia and fibula to the end of foot. The images were opened on Photoshop CS5 version 12 (Adobe Systems, Inc., San Jose, CA, USA) to be saved as Photoshop document files. Our interest was in the right-sided foot, beyond which exces- 
Table 1

Twenty-seven structures of the foot, which have been outlined and surface reconstructed

\begin{tabular}{lll}
\hline Systems & Groups & Structures \\
\hline Integumentary (1) & & Skin \\
\hline Muscular (24) & Retinaculum (3) & Inferior extensor retinaculum, flexor retinaculum, inferior fibular retinaculum \\
& Ankle joint (5) & Tibialis anterior, tibialis posterior, fibularis longus, fibularis brevis*, fibularis tertius \\
& Dorsum of foot (4) & Extensor hallucis longus*, extensor digitorum longus*, extensor hallucis brevis, extensor digitorum brevis \\
& 1st layer of sole (3) & Abductor hallucis*, flexor digitorum brevis, abductor digiti minimi \\
& 2nd layer of sole (3) & Flexor digitorum longus, quadratus plantae, lumbricalis \\
& 3rd layer of sole (4) & Flexor hallucis longus, flexor hallucis brevis, adductor hallucis, flexor digiti minimi brevis \\
& 4th layer of sole (2) & Plantar interosseus, dorsal interosseus \\
\hline $\operatorname{Vascular}(2)$ & & Dorsal venous arch, dorsal metatarsal vein
\end{tabular}

(Number of the structures). *Muscles, the belly and tendon of which are divided.

sive margins were cropped to reduce resolution $(3,040 \times$ $2,008)$ to $900 \times 720$; pixel size $0.2 \mathrm{~mm}$; color depth $24 \mathrm{bit}$; intervals $0.4 \mathrm{~mm}$ ).

\section{Segmentation}

We brought the already made segmented images, where the outlines of the skin and foot muscles had been drawn. ${ }^{[9,10]}$ The outlines, placed on the newly processed sectioned images, were manually edited and compensated to finish the full segmentation of the skin, muscles and cutaneous veins. Some muscles were divided into the muscle belly and tendon during delineation (Table 1). The outlines were then filled with different colors according to the individual components (Figure 1).

\section{Surface reconstruction}

Only color-filled outlines, excluding the sectioned images, were saved as bitmap (BMP) files. A total of 488 BMP files were placed in a Mimics file on Mimics version 10 (Materialise, Leuven, Belgium). Mimics utilized the color's brightness to recognize and cluster the serial outlines of every structure. After stacking the categorized colors, surface reconstruction of the whole structures was achieved at the same time. In each surface model, the accumulated outlines were removed and triangular surfaces were appropriately reduced in number. ${ }^{[1-14]}$ The surface models of individual components were painted with the already used colors. The whole models were rotated to make the dorsal, plantar, medial, and lateral views (Figures 2-5).
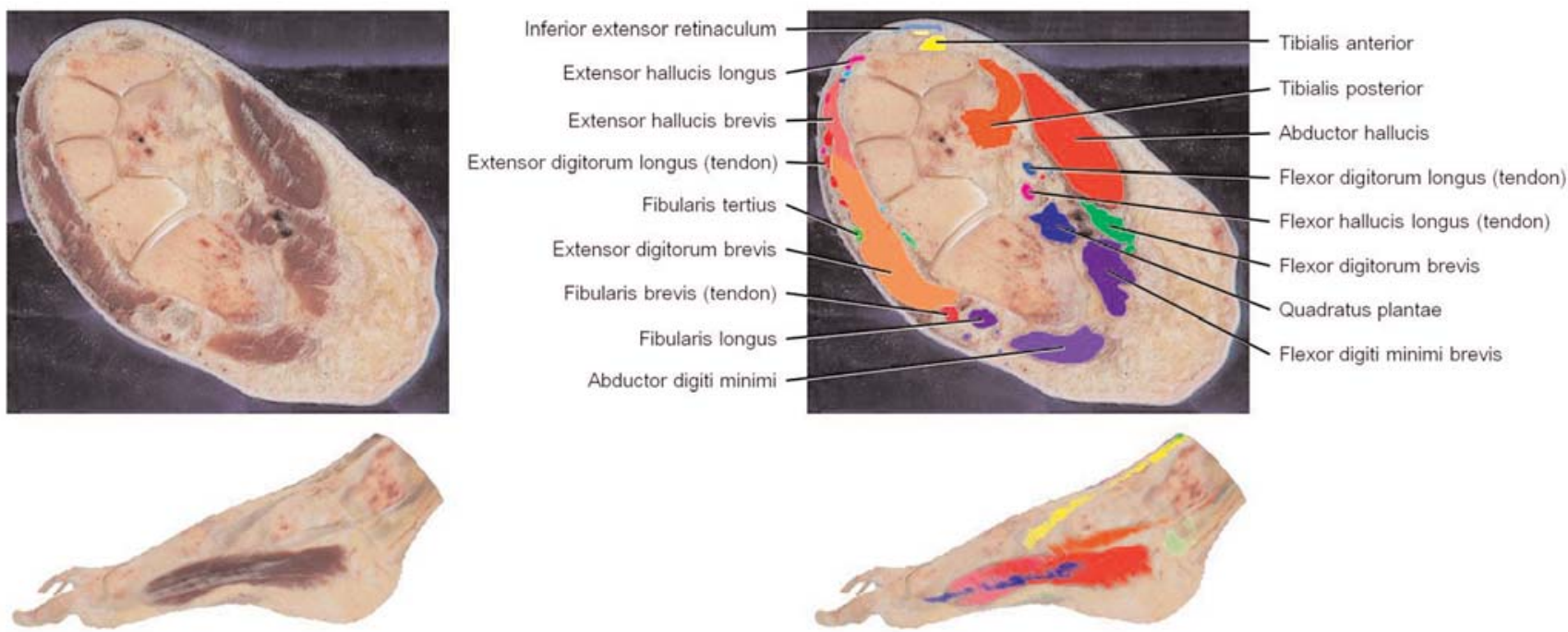

Figure 1. A sectioned image of the foot and the image with color-filled outlines (top row) and the resultant two stripped volume models (bottom row). 

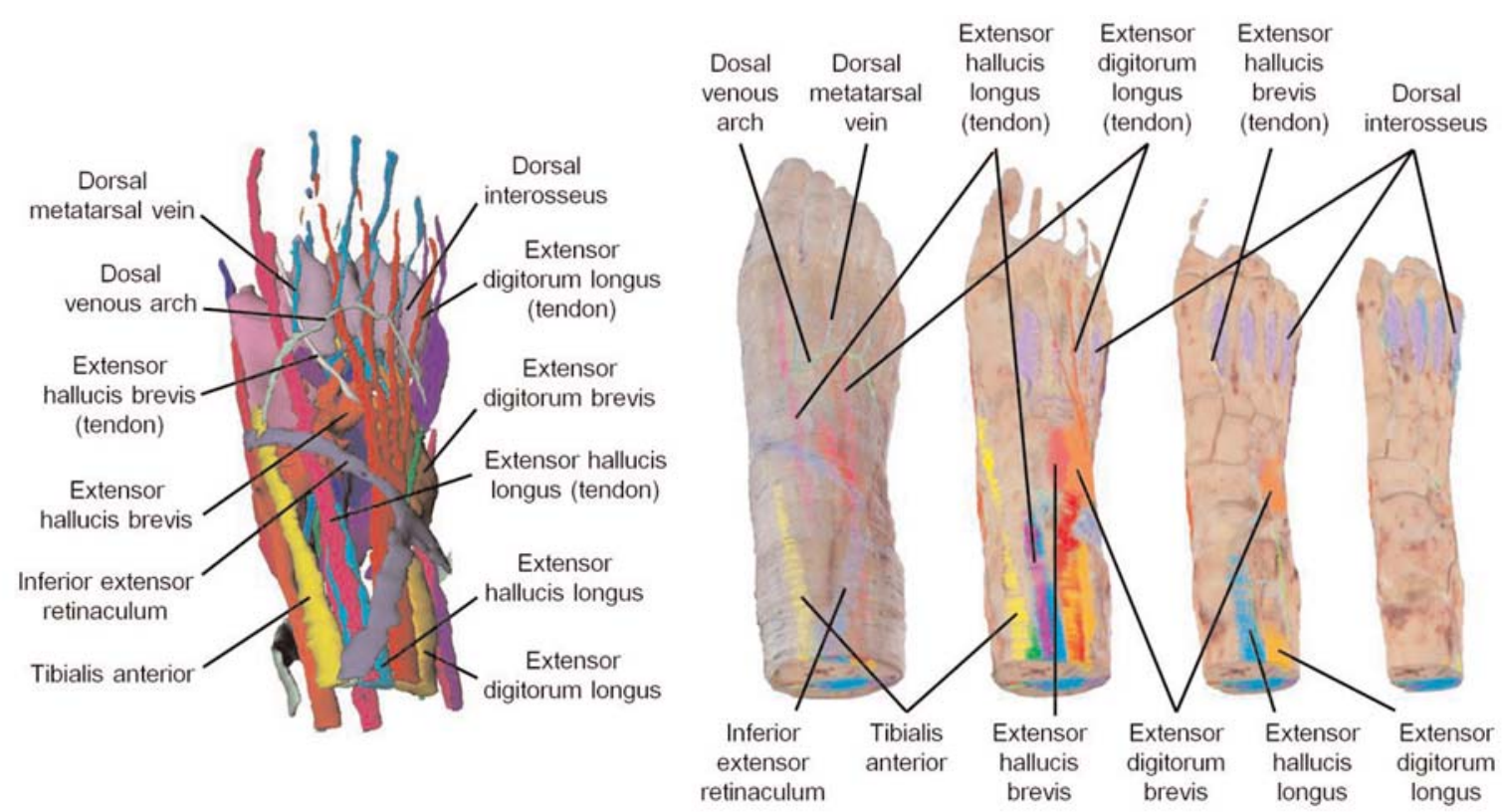

Figure 2. Surface models of the foot muscles (far left) and their volume model, gradually stripped (right four) in the dorsal view. Stripping thickness is $1 \mathrm{~mm}, 7 \mathrm{~mm}, 13 \mathrm{~mm}, 19 \mathrm{~mm}$ in that order.

\section{Volume reconstruction and exploration}

Due to the limitation of personal computer capacity, resolution of the sectioned images was reduced from $900 \times$ 720 (pixel size $0.2 \mathrm{~mm}$ ) to $300 \times 240$ (pixel size $0.6 \mathrm{~mm}$ ). The 488 sectioned images, where color-filled outlines were superimposed, were then utilized in volume reconstruction to build a volume model (voxel size $0.6 \mathrm{~mm}$ ).
We previously formulated and detailed the method to strip a volume model. ${ }^{[8]}$ Using the established method, the volume model of the foot was stripped at the distances, which denoted how far the voxels were from the skin. ${ }^{[15,16]}$ We decided the distances as $1 \mathrm{~mm}, 7 \mathrm{~mm}, 13 \mathrm{~mm}$, and 19 $\mathrm{mm}$ to acquire the stripped volumes demonstrating not only the sectioned images but also the outlined foot mus-
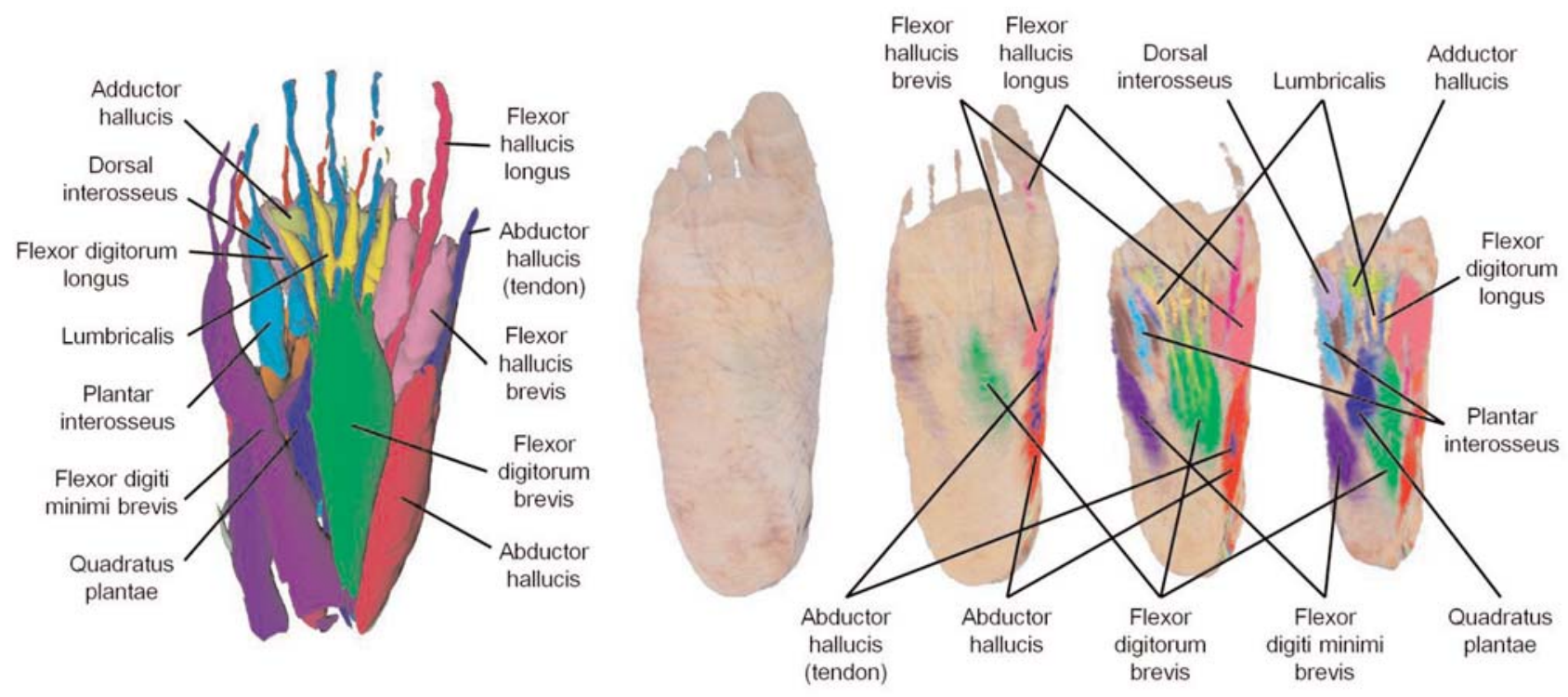

Figure 3. Surface models of the foot muscles (far left) and their volume model, gradually stripped (right four) in the plantar view. Stripping thicknesses are the same as in Fig. 2. 

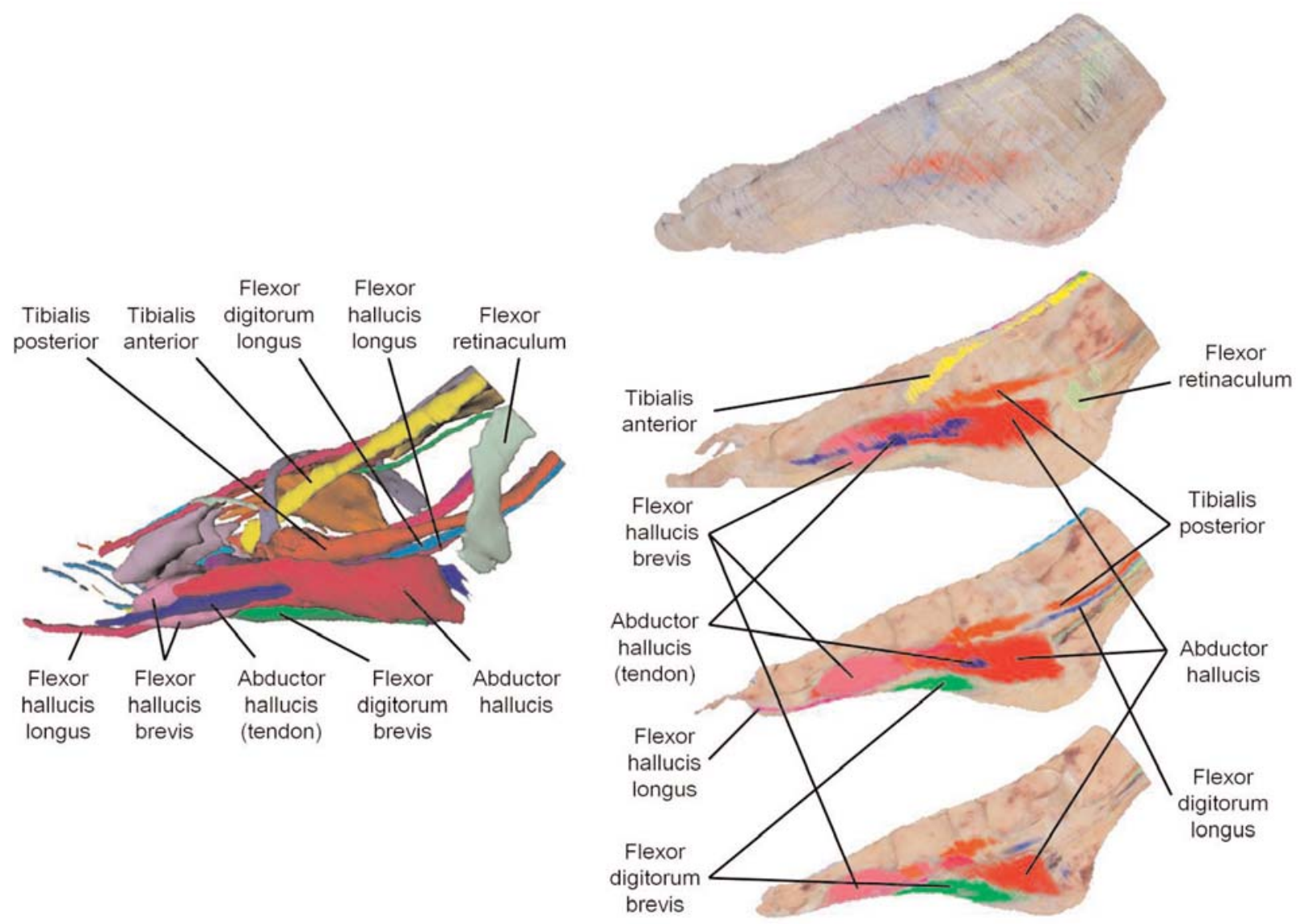

Figure 4. Surface models of the foot muscles (left) and their volume model, gradually stripped (right) in the medial view. Stripping thicknesses are the same as in Fig. 2.

cles in synthetic colors. We examined the volumes in the dorsal, plantar, medial, and lateral views together with the corresponding surface models (Figures 2-5).

\section{Results}

The file size of the 27 surface models was just $12 \mathrm{MB}$, whereas the volume model occupied $138 \mathrm{MB}$ in spite of the reduced resolution. Big data were due to the volume model consisting of about 35 million voxels. Nevertheless, visualization of the stripped volumes was not retarded in speed, once the outline-associated numbers were marked on the voxels of the volume model..$^{[8,17,18]}$

Tissue colors in the original sectioned images were consistent with those in the volume model (Figure 1). Moreover, allocated colors inside the outlines were the same in all surface models and stripped volume models. Therefore, morphological correlation of a set of stereoscopic models could be easily scrutinized by using the colors (Figures 1-5).
In the surface models, well-known muscles passing in three retinacula were verified. In the inferior extensor retinaculum, tibialis anterior, extensor hallucis longus, extensor digitorum longus, and fibularis tertius existed from the medial to the lateral sides (Figures 2 and 5). In the flexor retinaculum, the tibialis posterior, flexor digitorum longus, and flexor hallucis longus resided in sequence (Figure 4). In the inferior fibular retinaculum, fibularis longus and fibularis brevis were crossed (Figure 5). The result was same as they are known to position. ${ }^{[19]}$

When the volume model was stripped at $13 \mathrm{~mm}$, the tarsal bones and metatarsal bones were exposed in dorsal view (Figure 2). However, the bones were not shown in plantar view, even if it was stripped at $19 \mathrm{~mm}$ (Figure 3). The result was anticipated because of the prominently thick skin, subcutaneous tissue, and muscles of the sole.

Successive stripping revealed which muscles were closer to the skin. Among the three muscles passing the infe- 

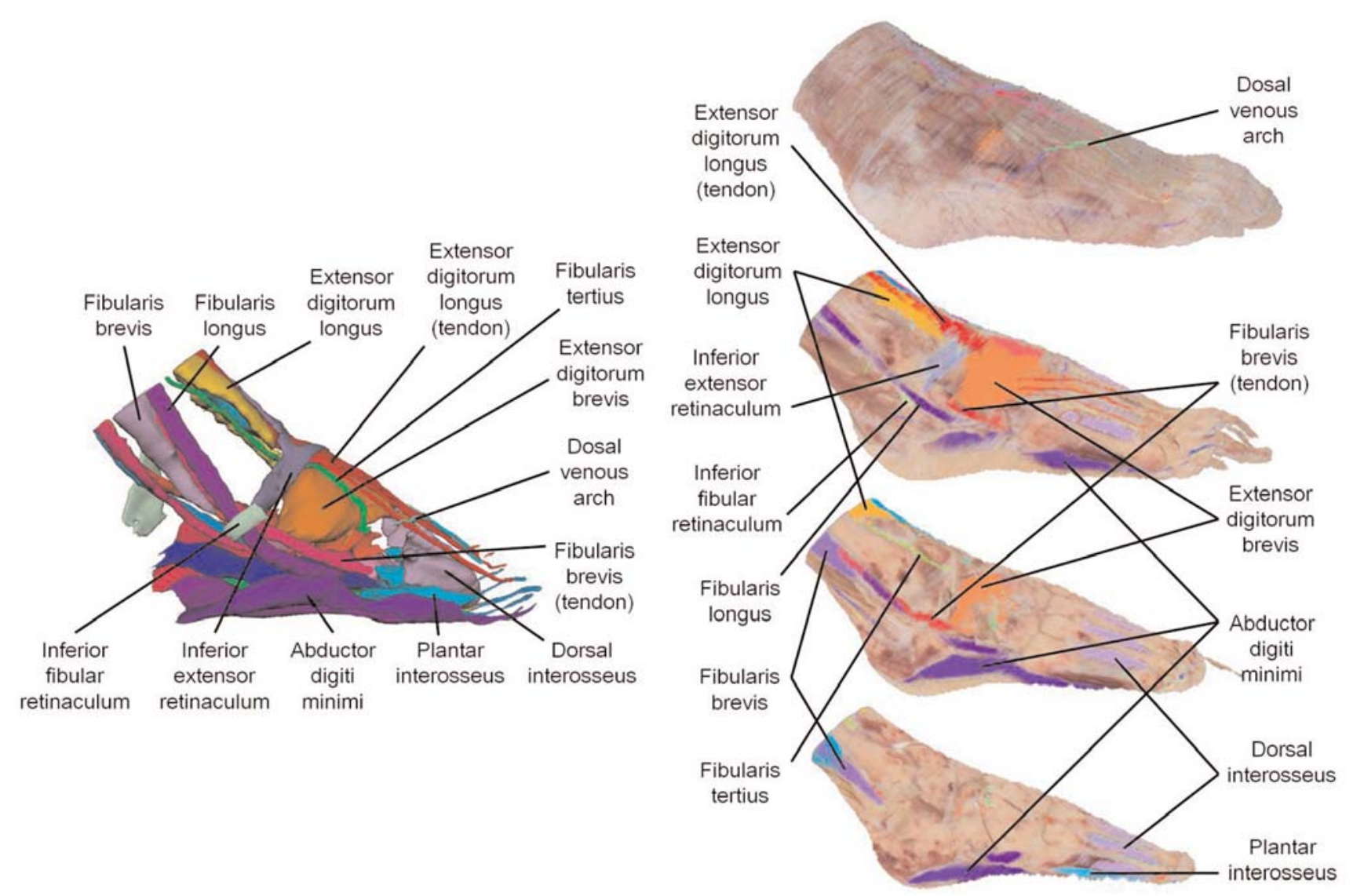

Figure 5. Surface models of the foot muscles (left) and their volume model, gradually stripped (right) in the lateral view. Stripping thicknesses are the same as in Fig. 2.

rior extensor retinaculum, the tibialis anterior was the most superficial, and then the extensor hallucis longus and extensor digitorum longus were in sequence. However, tendons, not muscle bellies, of the extensor hallucis longus and extensor digitorum longus were as superficial as the tibialis anterior (Figure 2).

In the 1st layer of the sole, the abductor hallucis was the most superficial, and the flexor digitorum brevis was the second. Thin skin and subcutaneous tissue in the medial side of the sole are not in contact with the ground because of the arch of the foot. Similarly, medial muscles of the 3rd layer that are flexor hallucis longus and flexor hallucis brevis were as superficial as the 2 nd layer that are flexor digitorum longus, quadratus plantae, and lumbricalis (Figure 3).

The stripped volumes showed that some muscles were wide since they were parallel to the skin. For instance, the abductor hallucis brevis and flexor hallucis brevis were flat muscles, which ran parallel with the medial skin of the foot (Figure 4).

\section{Discussion}

In this study, the volume model of foot was progressively stripped at constant intervals. The stripped volumes illustrate the depth of the foot muscles from the skin. Concerning a tubular structure, the stripped volumes would reveal the mural thickness and the uniformity of the thickness. Therefore, we already executed this exploration of the volume model in order to discern the morphological properties of the ascending colon wall. ${ }^{[8]}$

In order to apply this computer graphic method, two sets of image data are necessary. First one is the serial raw images that include the clinical images such as CTs and MRIs. Similarly, the serial slice images from the light or electron microscopy may be used to enlighten smaller histologic or cellular structures. Second one is 
the segmented images. The baseline of stripping (e.g., skin), at least, should be drawn either manually or automatically. The color-filled images and surface models, elaborated from the segmented images, are absolutely helpful in identifying the structures in the stripped volumes (Figures 1-5). ${ }^{[7]}$

In the case of the foot, the stripped volumes compensate the weak points of horizontal planes. The horizontal planes are literally determined in the anatomical position. During this standing posture, the skin and subcutaneous tissue of the sole are pressed, which is not consistent condition compared to the other body regions such as palm. The problem no longer exists, when the subject is laid down to acquire the CTs, MRIs in hospitals or the sectioned images in Visible Korean project. Instead, the plantar flexed foot causes difficult decision of the horizontal planes, which interferes with standardization. This research suggests the stripped volumes of the foot in supine subjects as a solution that keep the natural sole contour and yield standardized images regardless of the diverse degree of plantar flexion (Figure 3).

The Visible Korean research group also outlined and surface reconstructed the left foot muscles of the identical male cadaver from other inquiry. The surface models were contained in a portable document format (PDF) file, with which users are able to generate mixed displays of structures using the check boxes for their intentions. By manipulating the mouse, the surface models can be freely rotated, conveniently zoomed-in, zoomed-out, and shifted. ${ }^{[11-13]}$ The PDF file is downloadable on the Visible Korean homepage (anatomy.co.kr) free of charge; then, the surface models of the left foot can be compared with figures of the right foot in this paper.

All sectioned images, outlined images, and surface models of the bilateral feet are to be distributed to the interested researchers. Hopefully, the image data presented by authors can become a robust resource of the virtual dissection for medical students and the virtual operation for orthopedic surgeons. ${ }^{[20-24]}$

In the present study, the surface and volume models of the foot were built on the basis of sectioned images. The volume model was stepwise stripped to be examined with reference to the surface models. The exploration disclosed unfamiliar morphological aspect of the foot muscles. The innovative computer technology is expected to be applied to anatomical research on diverse regions.

\section{Acknowledgements}

This research was supported by Basic Science Research Program through the National Research Foundation of
Korea (NRF) funded by the Ministry of Education (grant number 2010-0009950). Raw data of the Visible Korean were acquired by the financial assistance from the Korea Institute of Science and Technology Information.

\section{References}

1. Park JS, Chung MS, Hwang SB, Lee YS, Har DH, Park HS. Visible Korean Human. Improved serially sectioned images of the entire body. IEEE Trans Med Imaging 2005;24:352-60.

2. Shin DS, Park JS, Shin BS, Chung MS. Surface models of the male urogenital organs built from the Visible Korean using popular software. Anat Cell Biol 2011;44:151-9.

3. Shin DS, Chung MS, Park JS. Systematized methods of surface reconstruction from the serial sectioned images of a cadaver head. J Craniofac Surg 2012;23:190-4.

4. Shin DS, Park JS, Chung MS. Three types of the serial segmented images suitable for surface reconstruction. Anat Cell Biol 2012; 45:128-35.

5. Park HS, Chung MS, Shin DS, Jung YW, Park JS. Accessible and informative sectioned images, color-coded images, and surface models of the ear. Anat Rec (Hoboken) 2013;296:1180-6.

6. Park JS, Jung YW, Lee JW, Shin DS, Chung MS, Riemer M, Handels H. Generating useful images for medical applications from the Visible Korean Human. Comput Methods Programs Biomed 2008;92:257-66.

7. Park JS, Chung MS, Chi JG, Park HS, Shin DS. Segmentation of cerebral gyri in the sectioned images by referring to volume model. J Korean Med Sci 2010;25:1710-5.

8. Shin DS, Chung MS, Shin BS, Kwon K. Laparoscopic and endoscopic exploration of the ascending colon wall based on a cadaver sectioned images. Anat Sci Int 2014;89:21-7.

9. Park JS, Chung MS, Hwang SB, Lee YS, Har DH. Technical report on semiautomatic segmentation using the Adobe Photoshop. J Digit Imaging 2005;18:333-43.

10. Shin DS, Park JS, Park HS, Hwang SB, Chung MS. Outlining of the detailed structures in sectioned images from Visible Korean. Surg Radiol Anat 2012;34:235-47.

11. Shin DS, Chung MS, Park JS, Park HS, Lee S, Moon YL, Jang HG. Portable document format file showing the surface models of cadaver whole body. J Korean Med Sci 2012;27:849-56.

12. Shin DS, Jang HG, Park JS, Park HS, Lee S, Chung MS. Accessible and informative sectioned images and surface models of a cadaver head. J Craniofac Surg 2012;23:1176-80.

13. Shin DS, Jang HG, Hwang SB, Har D-H, Moon YL, Chung MS. Two-dimensional sectioned images and three-dimensional surface models for learning the anatomy of female pelvis. Anat Sci Educ 2013;6:316-23.

14. Kim BC, Chung MS, Kim HJ, Park JS, Shin DS. Sectioned images and surface models of a cadaver for understanding the deep circumflex iliac artery flap. J Craniofac Surg 2014;25:626-9.

15. Pudney C. Distance-ordered homotopic thinning: a skeletonization algorithm for 3D digital images. Comput Vis Image Und 1998;72: 404-13.

16. Borgefors $\mathrm{G}$. On digital distance transforms in three dimensions. Comput Vis Image Und 1996;64:368-76. 
17. Krüger J, Westermann R. Acceleration techniques for GPU-based volume rendering. In: VIS'03: Proceedings of the 14th IEEE Visualization 2003 (VIS'03). Seattle (WA): IEEE Computer Society; 2003. p. 287-292.

18. Engel K, Hadwiger M, Kniss J, Rezk-Salama C, Weiskopf D. Realtime volume graphics. Natick (MA): AK Peters; 2006.

19. Moore KL, Dalley AF, Agur AMR. Clinically oriented anatomy. 7th ed. Philadelphia (PA): Lippincott Williams \& Wilkins; 2013.

20. Ackerman MJ. The Visible Human Project: a resource for education. Acad Med 1999;74:667-70.

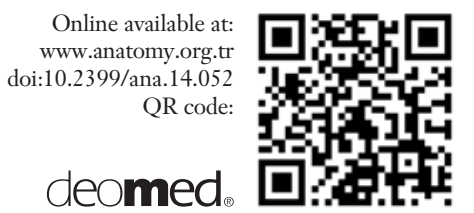

21. Jastrow H, Vollrath L. Teaching and learning gross anatomy using modern electronic media based on the Visible Human Project. Clin Anat 2003;16:44-54.

23. Heng PA, Zhang SX, Xie YM, Wong TT, Chui YP, Cheng CY. Photorealistic virtual anatomy based on Chinese Visible Human data. Clin Anat 2006;19:232-9.

24. Fang B, Wu Y, Li Y, Jin J, Tan L, Zhang S. Anatomical education and surgical simulation based on the Chinese Visible Human: a three-dimensional virtual model of the larynx region. Anat Sci Int 2013;88:254-8.

This is an open access article distributed under the terms of the Creative Commons Attribution-NonCommercial-NoDerivs 3.0 Unported (CC BY-NCND3.0) Licence (http://creativecommons.org/licenses/by-nc-nd/3.0/) which permits unrestricted noncommercial use, distribution, and reproduction in any medium, provided the original work is properly cited. Please cite this article as: Shin DS, Kwon K, Shin BS, Park HS, Lee S, Lee SB, Chung MS. Surface models and gradually stripped volume model to explore the foot muscles. Anatomy 2015;9(1):19-25. 